\title{
A Fractal Shape Signature
}

\author{
R I Taylor and P H Lewis \\ Department of Electronics and Computer Science \\ University of Southampton \\ Southampton, UK
}

\begin{abstract}
In this paper we describe a novel scheme for $2 \mathrm{D}$ shape representation based on fractal geometry. The representation (or fractal signature) of the shape is an external scalar transform which contains information from a wide range of scales. It appears to be unique except for size, orientation, position and reflection and we show that it degrades gracefully with noise. We show how the fractal signature can be used for fast shape matching and suggest how the technique can be extended to deal with partial boundaries.
\end{abstract}

\section{Introduction}

The analysis of two-dimensional curves is an important area in computer vision. For 2D shape matching we require a simple shape representation that can be easily compared to a library of known (or previously encountered) shapes. Most simple representations are deficient in the representation of either fine scale detail or in the description of larger scale global structure. In his review of shape coding techniques Marshall [6] states that the problems of scale have always been present in this area. Furthermore he states that working simultaneously over a range of scales must have more chance of achieving successful shape recognition than operating at a single arbitrary scale.

The main approaches to multi-scale description are Scale Space [3] and Fractal Geometry [5]. The latter approach produces descriptions which are simpler and yet, to quote Pentland [5], appear to capture all of the shape structure relevant to human perception.

\section{Fractional Brownian Model}

Figure 1 shows a closed boundary $\mathrm{B}$ which has a centroid C. Modeling the boundary as a 1-dimensional fractional Brownian function (see $[1,2,5]$ for details) we can define a function $\epsilon(\Delta \theta)$ as

$$
\epsilon(\Delta \theta)=E[|r(\theta+\Delta \theta)-r(\theta)|]
$$

The function $\epsilon(\Delta \theta)$ gives the expected value of the difference of the $r$ values for all pairs of points separated by an angle $\Delta \theta$. Note that $r(\theta)$ may be multivalued.

For a fractional Brownian function with zero-mean Gaussian distribution $N\left(0, \sigma^{2}\right)$ it can be shown that

$$
\epsilon(\Delta \theta) \cdot \Delta \theta^{-H}=K
$$




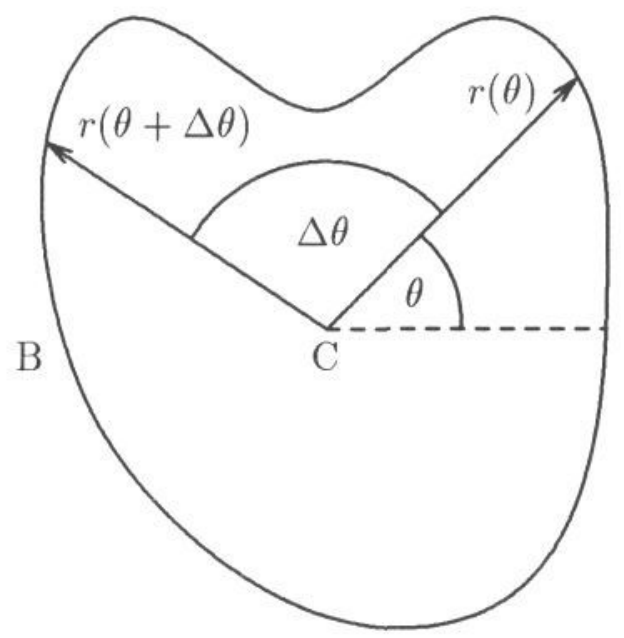

Figure 1: Arbitrary boundary

Where $K$ is a constant equal to $\sqrt{2 / \pi} \sigma$ and $H$ is a constant related to the fractal dimension $D$ of the curve by $H=2-D$.

Equation 2 is thus equivalent to

$$
\log \epsilon(\Delta \theta)-H \log \Delta \theta=\log K
$$

If we plot $\log \epsilon(\Delta \theta)$ against $\log \Delta \theta$ then we expect to get a straight line with a gradient $\mathrm{H}$ and intercept $\log K$.

\section{Shapes in Digital Images}

The above theory was derived in terms of a continuous boundary. When a region is extracted from an image we get a boundary that is made up of a list of discrete points. If we assume that the boundary is straight between points then we can scan the list and generate, by interpolating where necessary, a list of polar coordinates $\left(r_{i}, n_{i} \theta_{\min }\right) i=1,2, \ldots, N$ where $n_{i}$ is an integer and $\theta_{\min }$ is a constant. The value of $N$ will be $2 \pi / \theta_{\min }$ if the boundary function is single valued but greater if it is multi-valued.

We can then calculate $\epsilon(\Delta \theta)$ for integral multiples $k$ of $\theta_{\min }$ by scanning the polar list. As we are comparing all pairs of points this takes a time of $O\left(N^{2}\right)$.

$$
\epsilon\left(k \theta_{\min }\right)=\frac{\sum_{i, j} P(i, j)\left|r_{i}-r_{j}\right|}{\sum_{i, j} P(i, j)}
$$

where,

$$
P(i, j)= \begin{cases}1 & \text { if } n_{i}-n_{j}=k \\ 0 & \text { otherwise }\end{cases}
$$




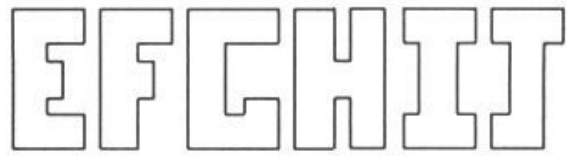

Figure 2: Various letters

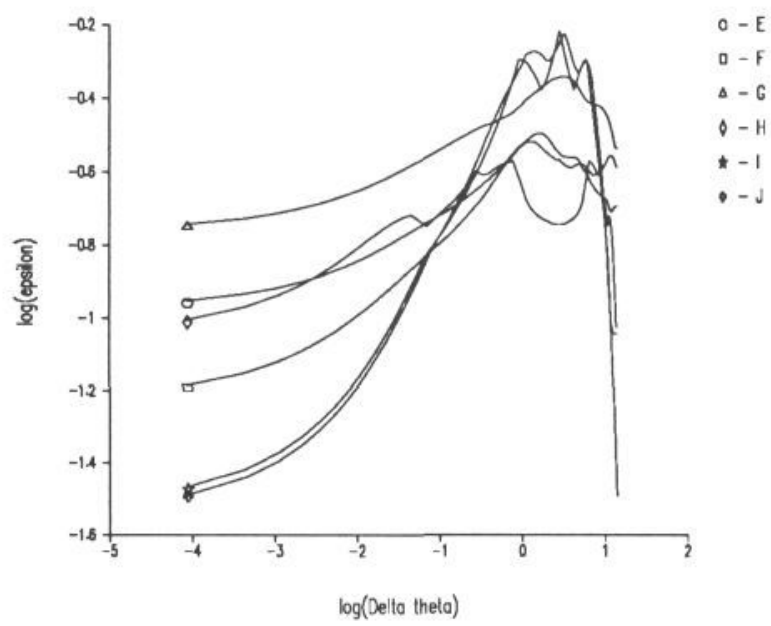

Figure 3: Fractal signatures of various letters

As $\epsilon(\Delta \theta)$ has period $2 \pi$ but is symmetrical about $\Delta \theta=\pi$ the range of $\Delta \theta$ is $\left[\theta_{\min } \ldots \pi\right]$. The value of $\theta_{\min }$ chosen was $\pi / 180$, giving 180 data points for the graph.

If we plot the log-log graphs for the shapes in figure 2 then we get the curves in figure 3. Linear regions occur where the shapes behave like fractals (ie. they are statistically self similar over a range of scales). The gradient of such sections has been used in relation to $3 \mathrm{D}$ image surfaces to estimate the fractal dimension as a measure of texture $[5,4,7,8]$. However, the curve as a whole represents a measure that changes continuously over all scales. So rather than use part of the curve, we use the whole curve as a multi-scale shape signature called the fractal signature.

\section{Invariance of Fractal Signatures}

Since $\epsilon(\Delta \theta)$ is measured relative to the centroid of the shape, the fractal signature is translation and rotation independent. As we use the difference of radii the signature is reflection invariant.

Scaling the shape would result in a similar scaling of the $\epsilon$ values and thus a constant offset on the log graph. In order to overcome this the signature can be normalised by dividing by the mean radius ie. we can define

$$
\epsilon^{\prime}(\Delta \theta)=\frac{E[|r(\theta+\Delta \theta)-r(\theta)|]}{E[r(\theta)]}
$$




\begin{tabular}{|l|rrrrrr|}
\hline & $\mathrm{E}$ & $\mathrm{F}$ & $\mathrm{G}$ & $\mathrm{H}$ & $\mathrm{I}$ & $\mathrm{J}$ \\
\hline $\mathrm{E}$ & 0 & 0.856 & 1.869 & 1.460 & 3.511 & 3.418 \\
$\mathrm{~F}$ & 0.856 & 0 & 2.387 & 1.599 & 3.072 & 3.032 \\
$\mathrm{G}$ & 1.869 & 2.387 & 0 & 2.888 & 3.424 & 3.098 \\
$\mathrm{H}$ & 1.460 & 1.599 & 2.888 & 0 & 3.840 & 4.015 \\
$\mathrm{I}$ & 3.511 & 3.072 & 3.424 & 3.840 & 0 & 0.958 \\
$\mathrm{~J}$ & 3.418 & 3.032 & 3.098 & 4.015 & 0.958 & 0 \\
\hline
\end{tabular}

Table 1: Euclidean Distance

\begin{tabular}{|l|rrrrrr|}
\hline & $\mathrm{E}$ & $\mathrm{F}$ & $\mathrm{G}$ & $\mathrm{H}$ & $\mathrm{I}$ & $\mathrm{J}$ \\
\hline $\mathrm{E}$ & 0 & 0.528 & 0.734 & 0.345 & 1.367 & 1.480 \\
$\mathrm{~F}$ & 0.528 & 0 & 1.242 & 0.832 & 0.894 & 1.012 \\
$\mathrm{G}$ & 0.734 & 1.242 & 0 & 0.656 & 1.714 & 1.799 \\
$\mathrm{H}$ & 0.345 & 0.832 & 0.656 & 0 & 1.634 & 1.748 \\
$\mathrm{I}$ & 1.367 & 0.894 & 1.714 & 1.634 & 0 & 0.147 \\
$\mathrm{~J}$ & 1.480 & 1.012 & 1.799 & 1.748 & 0.147 & 0 \\
\hline
\end{tabular}

Table 2: Bounded Area

This normalisation also cures the problem that the signatures of $r_{1}=f(\theta)$ and $r_{2}=f(\theta)+$ constant would have been the same.

The normalised signature appears to be unique except for scaling, rotation, translation and reflection. We are currently investigating the effects of other affine transforms (ie. stretching and shearing) on the signature of a shape. Preliminary results suggest that it may be possible to determine the signature of the transformed shape from the signature of the original. If this is so then it may also be possible, given an unknown shape, to determine whether it is an affine transformation of one of the known shapes.

\section{$5 \quad$ Matching Fractal Signatures}

Unknown shapes can be matched against a library of known shapes by comparing fractal signatures. Whilst the comparison of signatures is not trivial it should be much simpler than direct comparison of the boundaries since we have removed the effects of scaling, rotation, reflection and translation.

Figures 2 and 3 show the discriminating power of the signature. All the signatures are clearly distinct. The closest are those for the letters E and F, and $I$ and $J$, which is to be expected since the shapes are very similar.

So far we have used two different methods for comparing signatures

1. Euclidean Distance - treating the signatures as 180-dimensional vectors and calculating their separation.

2. Bounded Area - treating the signatures as continuous curves and calculating the (absolute) area between them.

The values of these metrics for the shapes in figure 2 can be seen in tables 1 and 2 . The former method appears to be the best since it gives relatively small 


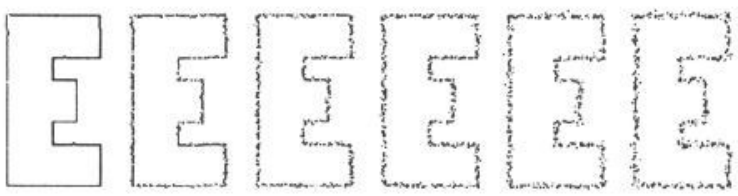

Figure 4: E with noise s.d. $0.2,0.4,0.6,0.8,1.0$ and 1.2

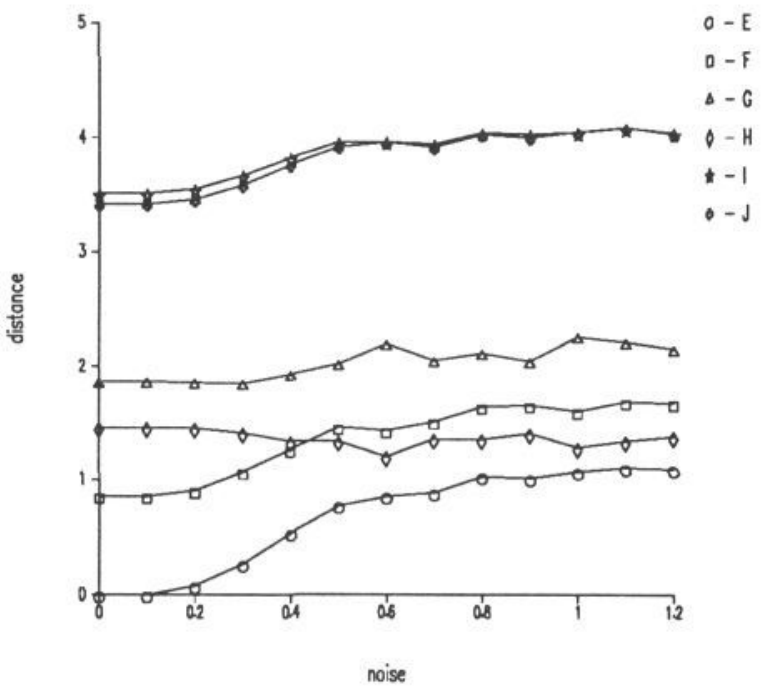

Figure 5: Euclidean metric on E's with noise

values for E-F and I-J whereas the latter method gives a smaller value for E-H than E-F.

\section{The Effects of Noise and Occlusion}

As each $\epsilon^{\prime}$ value is an average over the whole shape, minor occlusions and deformities are smoothed out to some extent. Thus, the signature degrades gracefully with occlusion and noise.

Figure 4 shows the E boundary (originally $65 \times 130$ pixels) with noise added to the $\mathrm{x}$ and $\mathrm{y}$ positions of all the points. The noise is zero-mean Gaussian with the given standard deviation and the coordinates rounded to the nearest integer. The noisy boundary is then considered to be the polygon joining the points in the same order in which they originally existed. Figure 5 shows the Euclidean distance between the signature of the noisy boundary and the signatures of the original shapes. The values plotted are an average for 10 examples (the distance can vary by about $5 \%$ ). For all the noisy shapes, the closest signature is that of the original E. However, for noise with a standard 


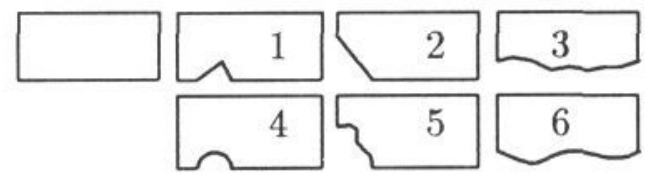

Figure 6: Rectangle occluded in various ways

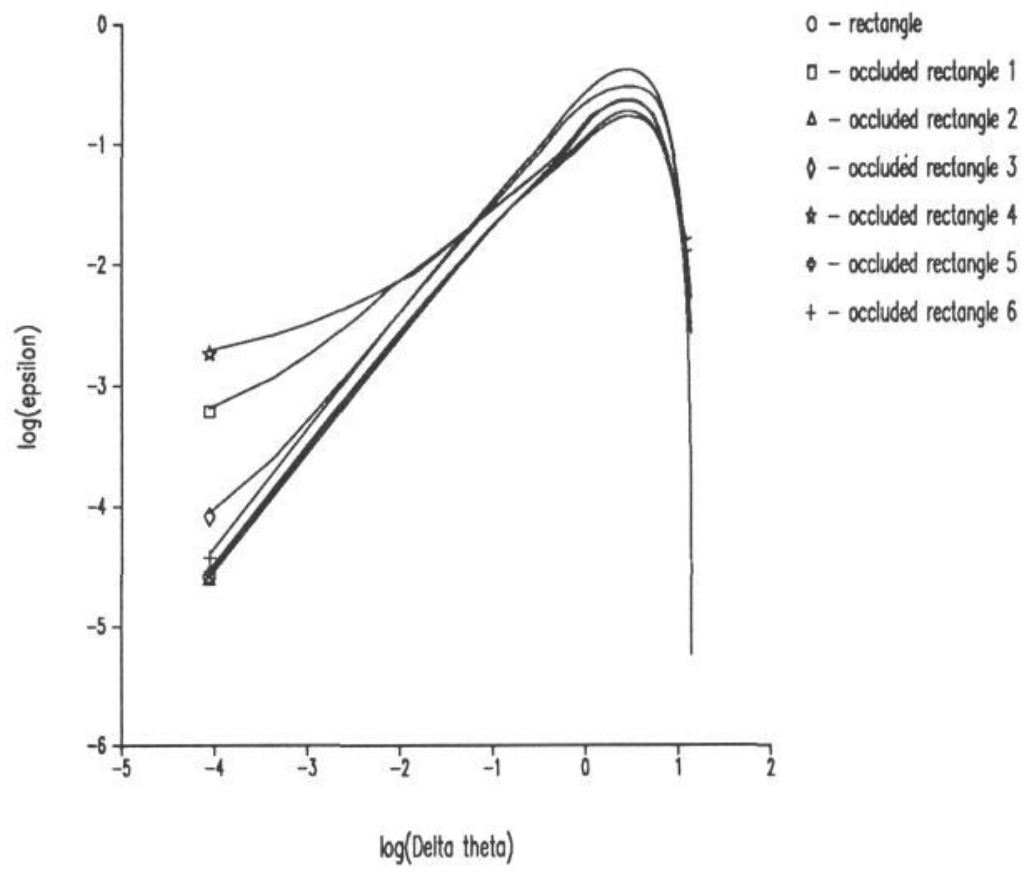

Figure 7: Fractal signatures of occluded rectangles

deviation of more than 0.6 pixels the distance to the $\mathrm{E}$ is greater than the distance between the original $\mathrm{E}$ and $\mathrm{F}$. These results, and others, suggest that two shapes whose signatures have a separation of less than 0.5 can be considered to be the same.

Figures 6 and 7 show how the signature of a rectangle changes when it is occluded in various ways. Some types of occlusion have more pronounced effects than others. What is important is the overall change in shape not the amount of the boundary occluded. Using the Euclidean metric described above the signatures of the occluded rectangles are at distances of between 4.6 and 5.6 from the original. Hence they are much too different to be recognised using the simple matching technique.

We are investigating the effects on the signature of removing parts of the shape boundary (and filling the gaps with straight segments). If the effects are predictable then it should be possible to identify parts of the boundary that may be due to an occlusion (from other image information) and remove them. This would then allow us to recognise partial boundaries. 


\section{Conclusions}

The proposed fractal shape signature has substantial potential for shape matching. Although it is simple, it contains information from a large range of scales. It appears to be unique except for size, orientation, position and reflection. In addition it degrades gracefully with noise. The major disadvantage with the present method is that although the signature degrades gracefully with occlusion, the matching method is too primitive to handle major occlusion. Hence we cannot generally recognise partial boundaries.

\section{Acknowledgment}

The work of the first author is supported by a SERC research studentship.

\section{References}

[1] B. B. Mandelbrot, Fractals: Form, Chance, and Dimension, Freeman, 1977.

[2] B. B. Mandelbrot, The Fractal Geometry Of Nature, Freeman, 1982.

[3] A. P. Witkin, Scale-space filtering, Proceedings of the 8th International Joint Conference on Artificial Intelligence, pp1019-1022, Karlsruhe, West Germany, 1983.

[4] G. G. Medioni and Y. Yasumoto, A note on using the fractal dimension for segmentation, Proceedings of the Workshop on Computer Vision: Representation and Control, pp25-30, Annapolis, Maryland, 1984.

[5] A. P. Pentland, Fractal-based description of natural scenes, IEEE Transactions on Pattern Analysis and Machine Intelligence, 6:661-674, 1984.

[6] S. Marshall, Review of shape coding techniques, Image and Vision Computing, 7:281-294, 1989.

[7] N Yokoya and K Yamamoto, Fractal-based analysis and interpolation of 3D natural surface shapes and their application to terrain modelling, Computer Vision, Graphics and Image Processing, 46:284-302, 1989.

[8] S S Chen and J M Keller and R M Crownover, Shape from fractal geometry, Artificial Intelligence, 43:199-218, 1990. 